\title{
Defining a Design Space for Persuasive Cooperative Interactions in Mobile Exertion Applications
}

\author{
Luís Duarte Paulo Ribeiro Tiago Guerreiro Luís Carriço \\ Departamento de Informática, Faculdade de Ciências da Universidade de Lisboa, \\ Edifício C6, Campo-Grande, 1749-016 Lisboa, Portugal \\ 1duarte@lasige.di.fc.ul.pt_pribeiro@lasige.di.fc.ul.pt

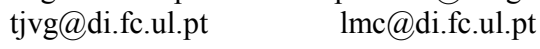

\begin{abstract}
This paper presents a design space for persuasive cooperative persuasive interactions for mobile exertion applications. This type of software bridges entertainment with workout activities, providing users with intuitive and fun ways to track their performances. Persuasion and, in particular, cooperative interactions play a pivotal role in user motivation - they are paramount to convince individuals to pursue their goals and overcome obstacles. Applications of this type have started to offer more diverse functionalities, often becoming difficult to label and pinpoint the type of design cues being employed to foster these persuasive and cooperative facets. In this article we propose a design space for this type of features in such software. The design exercise roots itself in existing literature and the results for an online survey we deployed to assess usage habits for this type of applications. Validation is underway and will be achieved via the creation of an application that covers the proposed dimensions.
\end{abstract}

Keywords: Mobile Exertion Applications, Design Space, Cooperation, Persuasion.

\section{Introduction}

Sedentary lifestyles are known to originate health conditions that could be prevented or even minimized by exercising a few hours a day [12][9][4]. The increasingly pervasive nature of work in our lives often inhibits individuals from spending a few minutes a day exercising, attempting to improve their physical and cognitive conditions. Unfortunately, many do not have the time to spend exercising, either because they are working late or because there are other matters that take that little time away. Other reasons for this non-existent exercising routine is the physical discomfort that comes associated with these particular kinds of activities [11] and lack of proactive partners to join in on the endeavour. The introduction of specialized mobile exertion applications (MEA) paved the way for users to engage more frequently in this type of activities.

In this paper we propose a design space for the inclusion for cooperative interactions in MEAs. We argue that cooperation is pivotal to engage users in this type of activities and, ultimately, lead them towards the goal of keeping in shape. The 
respectable number of products of this type available in different mobile application stores and the continuous interest of researchers in exploring this area testify its importance. Given the importance of cooperative play / interaction in the context of exercising, we considered to be of relevance to address existing caveats regarding the design of MEAs encompassing such features. To our knowledge, no research has attempted at consolidating the existing expertise about the role of cooperation and persuasion in MEAs into a design space. Our goal consists in providing a broad coverage of the dimensions related to persuasion and cooperation featured in MEAs.

\section{Related Work}

Our review of existing literature focuses on two relevant topics for this research. The first concerns the purpose and characteristics of MEAs, addressing their main features, usage scenarios and benefits. Lastly, we bridge the usage of persuasive technology with the concept cooperation, how they intertwine between each other and which types of cooperation are more commonly seen in the context of MEAs.

\section{Mobile Exertion Applications}

Mobile Exertion Applications (MEAs) are tools which translate into a set of effort systems assisting amateur and professional athletes during exercising activities. Endomondo Sports Tracker ${ }^{1}$, Nike ${ }^{2}$ or Runtastic ${ }^{3}$ are examples of such applications. Typically, these tools are used for hiking, jogging or cycling. By capitalizing on the ubiquitous nature and feature sets offered by modern smartphones such as GPS tracking or health sensor add-ons, developers and researchers envisioned the creation of these applications which aid users in tracking their exertion activities.

MEAs have a strong goal in granting users with physical benefit [6][12][13]. Factors like motivation, physical characteristics and experience contribute to the level of exertion [10]. At the same time, regular physical activity grants cognitive benefits such as improvements in mood, concentration and memory [4], as well as diminishing anxiety or stress [6][12][13]. Most MEAs offer a common set of functionalities: users are able to log their workout sessions for posterior usage as a comparison benchmark with future sessions. These are typically referred to as ghost recordings. Some MEAs also offer back-office tools allowing users to visualize their calories expenditure and performance variation over time.

\section{Role of Persuasion \& Cooperation}

Maximizing the physical and cognitive gain from these applications requires the user's commitment. Motivation emerges as a pivotal factor to entice users into pursuing these goals. Persuading individuals into engaging in physical activity has

\footnotetext{
${ }^{1}$ Endomondo: http://www.endomondo.com/

${ }^{2}$ Nike+: https://secure-nikeplus.nike.com/plus/

${ }^{3} \mathrm{https}$ ://www.runtastic.com/
} 
been the subject of study of designers and researchers alike [4]. Existing literature suggests some factors contributing positively towards the adoption of MEAs [3]. In the context of our research, we are focused on one particular aspect: how societal features are fostered as a persuasive factor in MEAs. B. J. Fogg has done extensive research in the domain of persuasion, often tying it with societal factors [5]. Existing research testifies the important of cooperation in user engagement and even performance [8]. Engaging in similar activities with friends or acquaintances has been shown to provide more pleasant experiences than when total strangers are involved. The impact of peer pressure in motivating groups of users is also paramount for the success of certain products and services. MEAs are no exception. Developers typically implement features which allow users to tackle each other's' challenges and compare the results between each other. More recently, the ability to monitor workout sessions live has also spawned new ways for groups of people to interact with each other and exert pressure to motivate each other.

\section{Mobile Exertion Applications' Usage Trends}

In order to properly create a design space we are required to go beyond the brief review of related literature about MEAs we presented. The first step in envisioning it encompassed deploying an online survey to extract information regarding how individuals utilize MEAs and their preferred setups while working out.

We established a set of goals which capitalized on the related literature we discussed and aimed at bridging the three key components of this research: MEAs, persuasion and cooperation. In the end our objectives consisted in:

- Identifying the most popular MEAs.

- Identifying the most regularly used features.

- Assessing how individuals utilize their MEAs with their peers.

- Assessing how individuals keep track of their progress prior, during and after each workout session.

We distributed an online questionnaire which was disseminated via mailing lists, social networks and direct request. A total of 194 participants responded to the call. Most subjects are young adults between 18 and 35 years old $(80 \%$ of the subject base), with a gender distribution of $56 \%$ males and $44 \%$ females.

\section{Technology Usage Characterization}

Smartphones were the most used mobile device by our participants (56\%). Tablets and basic mobile phones garnered the same percentage (21\% each).

In order to run mobile exertion products, these devices need to provide a few set of technologies. Most of these applications heavily use the devices' integrated GPS sensors and connections to the Internet. As such, we asked our participants if their devices provided these features. 136 participants stated that their devices provided them with GPS functionality (42\%). As for Internet connections, 150 participants stated that their devices had $3 \mathrm{G}$ telecommunication networks (46\%). 34 participants stated to not have access to any of these technologies (10\%). 123 participants stating 
their smartphone provided them with both GPS and 3G communication and 46 participants stating the same for their tablets.

\section{Workout Habits}

Most participants led a good relationship with exertion activities, with $51 \%$ reporting to at least exercise 2 or 3 times a week. $6 \%$ stated to only exercising at least once a month and $14 \%$ reported exercising sporadically. $8 \%$ stated to not exercise at all. $66 \%$ of the population spent between one and two hours in their workout sessions, while only $6 \%$ spent more than two hours on their workouts. The favoured sport practiced by the participants was running / jogging (38\%). As for the reasons behind their exercising, the indicated motivation was to "keep fit" (31\%), followed by "as a hobby" $(20 \%)$ and to "deal with stress" $(19 \%)$.

\section{MEA Usage}

When asked how familiar they were with MEA's, most participants stated to have no knowledge of them (38\%), while others knew about them but did not use them (32\%). Only 59 participants stated that they either have used them (16\%) or use them regularly (14\%). For these, Endomondo Sports Tracker, Nike+ and RunKeeper ${ }^{4}$ were their MEA's of choice. Some users preferred other non-mainstream applications such as Strava ${ }^{5}$, GetRunning ${ }^{6}$ or FitBit 7 .

Participants with MEA experience use these products because they display personal progress $(40 \%)$ and because they are able to motivate users to do better $(32 \%)$. Other reasons chosen by participants ranged from "It makes exercising more fun" $(15 \%)$, "It allows me to share my results with other people" $(8 \%)$ and "It allows me to compare my results with other people" (8\%), to more specific reasons such as "It allows me to keep a log of my exercises", "Allows me to see info about my tracks" and "Turns it into a game". For MEA power users, the main reason to use the software is the ability to motivate them (36\%), followed by the ability to show the users' progress $(34 \%)$. We were also interested in finding how often participants used MEA's. For those that stated to either use them regularly or to have used them, the typical frequency was of at least once a week (15\%).

\section{Motivation Catalysts}

We tried to assess the impact of motivational strategies which may or may not be present in MEA's, but which users recur to, prior to or during workout activities. For our purposes, we disregarded participants who stated to either having no knowledge of such strategies or that they have never used them before (effectively reducing the sample to 59 participants). We also looked into persuasive cues usage frequency. The following list covers the most common persuasive approaches:

\footnotetext{
${ }^{4}$ RunKeeper: http://runkeeper.com/

${ }^{5}$ Strava: http://www.strava.com/

${ }^{6}$ GetRunning: http://splendid-things.co.uk/getrunning/

${ }^{7}$ FitBit: http://www.fitbit.com/
} 
- Performance Messages - The presence of performance messages containing information about the user's progress made 57 participants feel more motivated (96\%). 27 participants reported to use this functionality frequently $(45 \%)$ and 17 stated to use it rarely $(29 \%)$.

- Music Players - Listening to music while working out is a very common feature used in mobile exertion products. From this study, 22 participants stated that using music player features made them feel more motivated $(38 \%) .63 \%$ of the users use persuasive music features frequently.

\section{Role of Peers}

As far as social habits are concerned, $42 \%$ of the participants stated to exercise by themselves, while $32 \%$ stated to doing it in the company of a friend or colleague and $26 \%$ within a group.

A second facet of how peers interact between each other concerns the momentum they build before (arranging for the meeting), during (observing each other and cheering for one another) and after (commenting on how good / bad their performance was) the workout session. Since modern MEAs often give support to these features we questioned users about their opinions, reaching the following results:

- Competing against yourself - The existence of competition against oneself proved to be the feature with higher positive influence on the participants' motivation. From the 33 participants that replied to this strategy having some influence on their motivation, 78\% stated they felt more motivated. Of these, $49 \%$ compete against themselves frequently. Competition against oneself is a natural benchmark for simple performance comparisons. MEA's often call this feature "ghost" mode since a user is challenging a "ghost" recording of him / herself or of another person in the past, enhancing the importance of cooperation to improve one's condition.

- Competing against others - 28 participants responded they use another person as a benchmark for their workout activities and it has some influence on their motivation. $51 \%$ of these participants perceive this strategy as being a positive persuasive approach, increasing their motivation. Of these, only $28 \%$ recurred to this strategy with some frequency.

\section{A Design Space for Cooperative Mobile Exertion Applications}

In light of the review of relevant literature in this domain and the results obtained from the online questionnaire we distributed, we envisioned a design space for MEAs with a focus on the cooperative aspect of these applications and related activities. The design space encompasses a set of dimensions and categories which are used to foster cooperative and persuasive interactions between the individual working out and other individuals whether they also participate in the session directly (i.e. other athletes) or not (i.e. audiences). 


\section{Activities Overview}

As pointed in the questionnaire's results, in the context of exertion activities and, in particular, in the usage of MEAs, persuading via cooperation may be present in events other than the workout session itself, among which:

- Workout session arrangement \& anticipation - users often pre-emptively arrange for the workout session establishing where it will take place at what time and the duration.

- Workout session discussion - after a workout session users also often use social networks or dedicated MEA software to share their accomplishments and their workout performance evolution.

These two activities which take place outside the duration of the workout session possesses a common dimension - time, with one occurring before the session and the latter after it. During the exertion session itself, one can find other relations:

- Exercising with a human peer - this is a type of activity which does not necessarily require a MEA to come to fruition (although it can be enhanced by one). One can also state that this type of activity requires the intervenients to perform at the same time and at the same location for it to make sense.

- Exercising with a virtual peer - on the other side of the spectrum we can encounter a type of event which is possible due to the usage of MEAs, consisting in working out with virtual peers. The transition from real to virtual peers introduces new possibilities. These ghost recordings can contemplate sessions of users who performed that activity in the same location in which the individual is exercising or a totally different one.

\section{Dimensions \& Categories}

The previous exercise allowed us to pinpoint a set of activities and scenarios which tie the primary concepts retrieved in our questionnaire. We can now materialize them formally in a design space for the usage of cooperative persuasion in MEAs. We establish the exertion activity (and intrinsically the user) to be at the centre of the design space referential.

Stakeholders. This dimension addresses the individuals involved in the exertion activity as a whole. Other individuals may intervene at different stages of the activity. In light of this frame, we can define the following stakeholders:

- Spectators - this entity assumes an indirect influence on the user, since it is not participating directly in the exertion activity itself. Spectators often interact with the user through social networks or, if supported, via the MEA.

- Partner - one of the greatest sources of motivation is someone or something one can compare him / herself to. The existence of a partner can be a driving force behind one's motivation during exertion activities. The user may be persuaded by partners exercising in the same instant or be challenged by "ghosts" of those opponents. 
- Self - the last category addresses scenarios in which users cooperate with themselves to improve their motivation. This can only be carried out if one possesses past data of a workout session - a ghost recording of that user.

Space. The characteristics of the exertion activity are also an important part of the design space. The first we address is tied with the location of the exertion activity. Cooperation plays a role here, especially if we take into account the possibility of adding either real or virtual partners. As such, the space dimension contemplates the following categories:

- Co-Located - addresses activities in which the user and his / her partners exercise in the same location.

- Distributed - pertains to those activities in which a user may be performing at a determined location, while others are doing it in a totally different place.

Time. Accompanying the previous dimensions we introduce the time dimension. In this overview, we will tie-in most of the already described dimensions and categories along with emphasizing aspects related with the role of cooperation in the persuasive process behind the MEA. Time is divided in three categories:

- Before - the user can be persuaded via events which took place before the activity he / she is engaged in: a ghost recording is a log of a session which took place previously but may be in use during the current session; being influenced by partners prior to the session in a discussion.

- During - during the session, there are two scenarios in which cooperation plays a role in user motivation: a co-located and a geographically distributed one. In the first, users can keep track of each other's performance visually, while in the latter other communication channels are required to be supported by the MEA (audio for instance).

- After - persuasion based on cooperative efforts after the exertion activity can serve two purposes which are linked together. First it can be used as a source of analysis for the user. Secondly, it serves as a build-up catalyst for the next exertion session.

Persuasive Feedback. Here, two sub-dimensions emerge: the first pertains to the scope of the feedback and the second to the instruments used to convey it. Scope can be easily defined: feedback may privately address the user or publicly target all partners partaking in an exertion session.

Our review of existing literature allowed us to categorize feedback according to specific parameters. We found that users often receive feedback in the form of written or audio performance messages (e.g. "you're going faster"). However, some mentioned the usage of music or cheering sounds as a way to motivate themselves. As such, we can define these vehicles according to the following couple of categories:

- Natural Language - this type of feedback is capable of being naturally and easily comprehended by human beings without requiring intensive interpretation and with no second meaning involved.

- Metaphorical Content - this type of content encompasses messages which are not directly understandable but may be interpreted in a way that delivers a determined message to the user. 


\section{Conclusions \& Future Work}

This article presented a design space for cooperative persuasion interactions in mobile exertion applications. Ensuring a sustainable and healthy lifestyle is the aim of several applications available for smart-phones. MEAs attempt to materialize this via persuading their users into exercising more often. Like in other areas, sharing these endeavours with partners and acquaintances can be mutually beneficial, aiding both parties in improving their condition. Validation of this design space in under way, with the development of a MEA which contemplates the presented dimensions.

\section{References}

1. Adams, M. et al (2009). A Theory-based Framework for Evaluating Exergames as Persuasive Technology. In Proceedings of the 4th International Conference on Persuasive Technology.

2. Chittaro, L. et al (2012). Turning the classic Snake mobile game into a location-based exergame that encourages walking. In Proceedings of the 7th International Conference on Persuasive Technology (PERSUASIVE, 2012).

3. Chittaro, L. et al. 2013. Exploring audio storytelling in mobile exergames to affect the perception of physical exercise. In Proceedings of the 7th International Conference on Pervasive Computing Technologies for Healthcare (PervasiveHealth '13). ICST, Brussels, Belgium, Belgium, 1-8.

4. Gao, Y. et al (2012). The Acute Cognitive Benefits of Casual Exergame Play. In Proceedings of the SIGCHI Conference on Human Factors in Computing Systems (CHI, 2012). ACM, New York.

5. Fogg, BJ. (2002). Persuasive Technology: Using Computers to Change What We Think and Do. In Ubiquity Magazine.

6. Gerling, K. et al (2012). Full-body Motion-based Game Interaction for Older Adults. In Proceedings of the SIGCHI Conference on Human Factors in Computing Systems (CHI 2012). ACM, New York.

7. Macvean, A. et al (2012). iFitQuest: A School Based Study of a Mobile Location-Aware Exergame for Adolescents. In Proceedings of the 14th International Conference on HumanComputer Interaction with Mobile Devices and Services (MobileHCI, 2012).

8. Mandryk, R. et al. Physiological indicators for the evaluation of co-located collaborative play. In Procs of CSCW 2004.

9. Mueller, F., 2009. Exertion in Networked Games. In Proceedings of the 4th International Conference on Foundation of Digital Games (FDG, 2009). ACM, New York.

10.Mueller, F. et al (2010). Jogging over a distance between Europe and Australia. In Proceedings of the 23nd annual ACM symposium on User interface software and technology (UIST '10). ACM, New York, NY, USA, 189-198.

11.Mueller, F. et al (2011). Designing Sports: A Framework for exertion Games. In Proceedings of the SIGCHI Conference on Human Factors in Computing Systems.

12.Weinberg, et al (2006). Foundations of Sport and Exercise Psychology. Human Kinetics.

13. Whitehead, A. et al (2010). Exergame Effectiveness: What the Numbers Can Tell Us. In Proceedings of the 5th ACM SIGGRAPH Symposium on Video Games.

14.Calories burned while jogging: http://www.myfitnesspal.com/topics/show/134478-accurateformula-to-determine-calories-burned-jogging 